ARTICLE

DOI: $10.1038 / s 41467-018-06279-x$

\title{
Zigzag carbon as efficient and stable oxygen reduction electrocatalyst for proton exchange membrane fuel cells
}

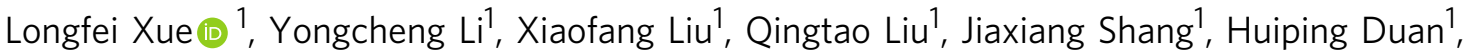 \\ Liming Dai ${ }^{2,3} \&$ Jianglan Shui ${ }^{1}$
}

Non-precious-metal or metal-free catalysts with stability are desirable but challenging for proton exchange membrane fuel cells. Here we partially unzip a multiwall carbon nanotube to synthesize zigzag-edged graphene nanoribbons with a carbon nanotube backbone for electrocatalysis of oxygen reduction in proton exchange membrane fuel cells. Zigzag carbon exhibits a peak areal power density of $0.161 \mathrm{~W} \mathrm{~cm}^{-2}$ and a peak mass power density of 520 $\mathrm{W} \mathrm{g}^{-1}$, superior to most non-precious-metal electrocatalysts. Notably, the stability of zigzag carbon is improved in comparison with a representative iron-nitrogen-carbon catalyst in a fuel cell with hydrogen/oxygen gases at $0.5 \mathrm{~V}$. Density functional theory calculation coupled with experimentation reveal that a zigzag carbon atom is the most active site for oxygen reduction among several types of carbon defects on graphene nanoribbons in acid electrolyte. This work demonstrates that zigzag carbon is a promising electrocatalyst for low-cost and durable proton exchange membrane fuel cells.

\footnotetext{
${ }^{1}$ School of Materials Science and Engineering, Beihang University, No. 37 Xueyuan Road, Beijing 100083, China. ${ }^{2}$ Department of Macromolecular Science and Engineering, Case Western Reserve University, 10900 Euclid Avenue, Cleveland, OH 44106, USA. ${ }^{3}$ UNSW-CWRU International Joint Laboratory, School of Chemical Engineering, University of New South Wales, Sydney, NSW 2051, Australia. Correspondence and requests for materials should be addressed to H.D. (email: hpduan@buaa.edu.cn) or to L.D. (email: liming.dai@case.edu) or to J.S. (email: shuijianglan@buaa.edu.cn)
} 
roton exchange membrane fuel cells (PEMFCs) attract worldwide attention because they can efficiently convert hydrogen energy into electricity. The device uses an acid solid electrolyte (sulfonated tetrafluoroethylene-based fluoropolymer-copolymer) and is the most mature type of fuel cell. In the PEMFC, the oxygen reduction reaction (ORR) is the ratedetermining step. Noble metal platinum is generally employed as a catalyst, and has hindered the wide application of PEMFCs. To solve this problem, a variety of non-precious metal catalysts (NPMCs), including nitrogen-coordinated transition metals $(\mathrm{M}-\mathrm{N}-\mathrm{C}, \mathrm{M}=\mathrm{Fe}, \mathrm{Co} \text {, etc. })^{1-7}$, and even metal-free catalysts (mainly heteroatom-doped carbon nanomaterials) have been developed $^{8-12}$. The NPMCs present satisfactory activity and stability during half-cell characterization (a three-electrode system) with both alkaline and acidic electrolytes. However, poor stability in the actual PEMFC device is still one of the biggest challenges for NPMCs, especially for M-N-C electrocatalysts ${ }^{13-15}$. Recently, an $\mathrm{N}$-doped graphene/carbon nanotube (CNT) composite has been reported to exhibit a stable PEMFC performance, though at a relatively low activity, which attracted a great deal of interest in metal-free electrocatalysts for the PEMFCs ${ }^{16}$.

In addition to the heteroatom-doped carbon nanomaterials, recent studies demonstrated that the defect-rich carbon could also effectively catalyze ORR in both alkaline and acidic electrolytes $^{17-21}$. Thus, graphene nanoribbons (GNRs) could be promising metal-free catalysts due to the large aspect ratio and numerous defects along their edges 22,23 . GNRs with different edge structures can be obtained by unzipping CNTs in different ways $^{24-27}$. In particular, Tour et al. created a zigzag-type edge on unzipped multiwall carbon nanotubes (MWCNTs) using $\mathrm{H}_{2} \mathrm{SO}_{4}$ and $\mathrm{KMnO}_{4}{ }^{25,28}$. This offers an opportunity to study the specific activity of a certain type of carbon defects. In spite of the abundant defect sites, GNRs have not been characterized with any decent ORR activities in the acid half cell. The easy restacking of GNRs could lead to low activity since most of the GNR edges in the restacked materials are blocked from the reactants, leading to extremely low utilization of the active sites. This blocking effect will be more serious in a PEMFC because the catalyst layer in a PEMFC is much thicker than that in a half cell. Furthermore, in contrast to the liquid electrolyte in a half cell, the solid ionomer electrolyte in PEMFCs causes a severe mass transport barrier. So far, the promise of GNRs (or graphitic carbon defects) for electrocatalysis in PEMFCs is yet to be realized.

Here a composite of zigzag-edged GNRs on CNTs (GNR@CNT) is synthesized for use as the cathode catalyst in $\mathrm{H}_{2}$ / $\mathrm{O}_{2}$ PEMFCs. The CNT backbones in the middle of GNR, coupled with a carbon black spacer, effectively exposes the zigzag carbon for ORR. Compared with previously reported metal-free electrocatalysts ${ }^{16}$, GNR@CNT delivers an unprecedented peak power density of $520 \mathrm{~W} \mathrm{~g}^{-1}$ in PEMFCs, even better than that of the $\mathrm{N}$ doped counterpart. More importantly, CNT@GNR presents a remarkable stability in PEMFCs. A density functional theory (DFT) calculation reveals zigzag carbon is indeed the active site on the GNR.

\section{Results}

Fabrication and characterization of catalysts. Figure 1 schematically illustrates the partial unzipping of a MWCNT into zigzag-edged GNR with a CNT backbone. Following the published method ${ }^{25}$, purified commercial MWCNTs (diameters $\sim 20$ $\mathrm{nm}$ and lengths of $0.5-2 \mu \mathrm{m}$ ) were partially unzipped by concentrated sulfuric acid and potassium permanganate, forming an oxidized GNR@CNT hybrid (Fig. 1b). As shown in

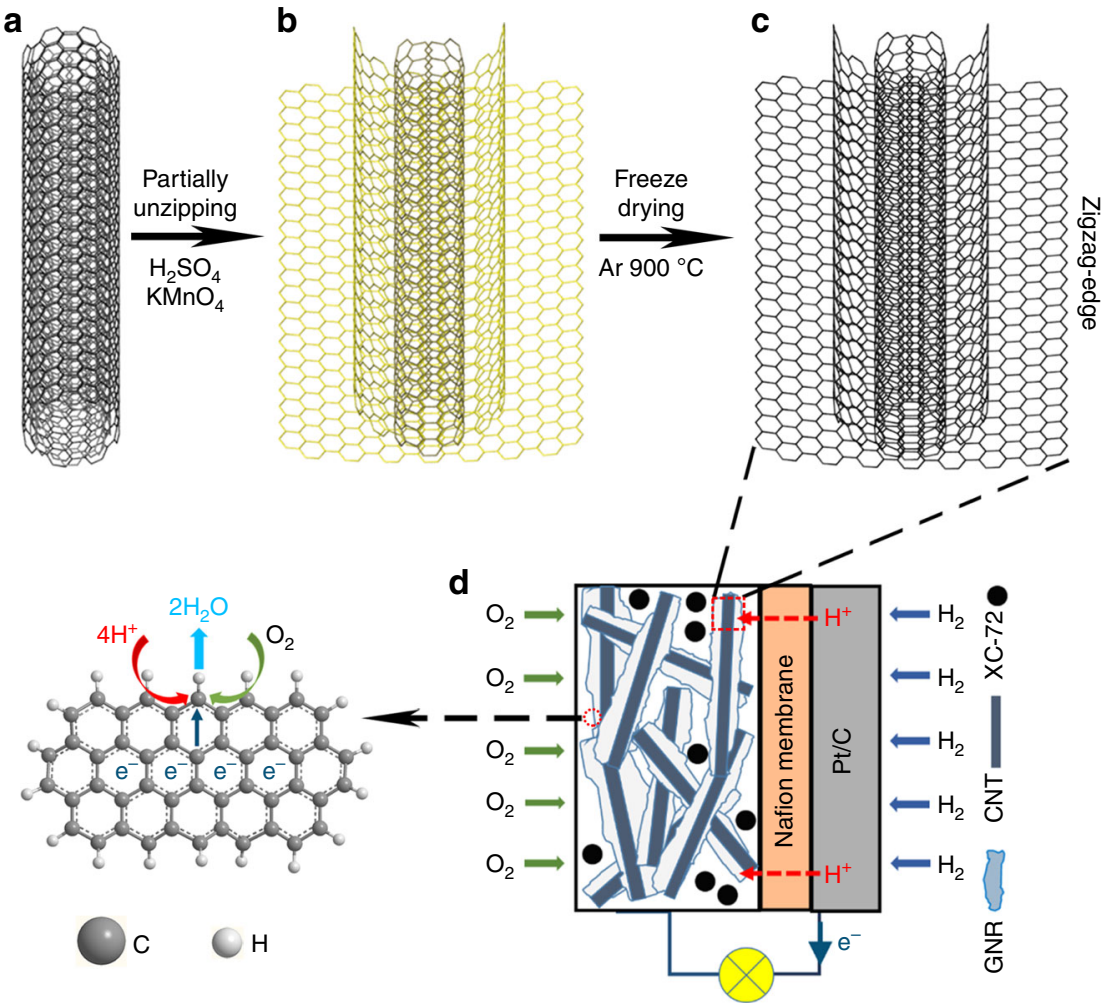

Fig. 1 Schematic illustration. The synthetic route of zigzag-type graphene nanoribbons on carbon nanotubes (GNR@CNT) from a MWCNT to b partially unzipped oxidized CNT and to c GNR@CNT. d The application as oxygen reduction reaction catalyst in a proton exchange membrane fuel cell (PEMFC). Carbon black XC-72 is used as spacer to prevent the stacking of active materials 
Supplementary Fig. 1a, b, the oxidized GNR has a width of approximately $60 \mathrm{~nm}$ and is attached on the CNT backbone. Finally, the oxidized GNR was reduced at high temperature, producing a GNR@CNT composite. According to previous reports ${ }^{25,28}$, this unzipping method created zigzag-type carbon along GNR edges. GNR@CNT thus obtained was then used as the cathode catalyst in a PEMFC. The rigid CNT backbone could prevent the stacking of GNR in the catalyst layer, as schematically shown in Fig. 1d. Meanwhile, carbon black was introduced into the catalyst layer to further separate GNR@CNT, facilitating the mass transport in the catalyst layer to ensure a high utilization of zigzag carbon defects on the GNR edges ${ }^{16}$. As a result, GNR@CNT exhibited efficient and stable ORR catalytic activity in PEMFCs, as discussed below. For comparison, three control samples have been prepared, including a completely unzipped MWCNT (denoted GNR) using a double-concentrated $\mathrm{KMnO}_{4}$ than that for GNR@CNT, and the N-doped counterparts (NGNR@CNT and N-GNR) by annealing corresponding materials in $\mathrm{NH}_{3}$ at $800^{\circ} \mathrm{C}^{29}$.

Figure 2 and Supplementary Fig. 2 compared the morphology of GNR@CNT, GNR, and their N-doped counterparts. As shown in Fig. 2a and Supplementary Fig. 2a, the partially unzipped CNTs formed ribbon-like graphene nanosheets closely attached on the remaining CNT backbones. $\mathrm{NH}_{3}$-treatment could severely corrode the GNR, leaving the CNT skeleton as the dominant material, as observed in Fig. 2c and Supplementary Fig. 2b. Fourier transform infrared (FT-IR) spectra of GNR@CNT and NGNR@CNT suggested the reduction of oxidized GNR after Ar and $\mathrm{NH}_{3}$ annealing (Supplementary Fig. 3), showing the removal of oxygen functional groups. Different from partially unzipped CNTs, the fully unzipped CNTs displayed a typical graphene-like morphology without any residual CNTs (Fig. 2e and Supplementary Fig. 2c). Meanwhile, $\mathrm{NH}_{3}$ corrosion created dense holes with diameter of $5-10 \mathrm{~nm}$ on N-GNR sheets (Fig. $2 \mathrm{~g}$ and Supplementary Fig. 2d). The surface composition of GNR@CNT and N-GNR@CNT were analyzed by X-ray photoelectron spectroscopy (XPS). The survey scan did not detect any $\mathrm{Ni}$ signals (catalyst for the CNT growth) after the acid pretreatment (Supplementary Fig. 4a). The high-resolution C $1 s$ spectrum in Supplementary Fig. $4 \mathrm{~b}$ reveals the dominance of $s p^{2}$ carbon, suggesting a highly graphitic structure of GNR@CNT. The XPS spectra of N-GNR@CNT indicates a successful doping of 3.09 at. $\% \mathrm{~N}$, which was composed of pyridinic $\mathrm{N}(398.5 \mathrm{eV})$, pyrrolic $\mathrm{N}$ $(399.8 \mathrm{eV})$, quaternary $\mathrm{N}(401.2 \mathrm{eV})$, and intercalated nitrogen molecules and/or oxides (403.5 eV) (Supplementary Fig. 5).

Electrochemical performance evaluation. The electrocatalytic activity of the resultant catalysts was first thoroughly investigated in a half cell. The optimal annealing temperatures of $900^{\circ} \mathrm{C}$ for GNR@CNT in Ar and $800^{\circ} \mathrm{C}$ for N-GNR@CNT in $\mathrm{NH}_{3}$ were deduced by comparing the linear sweep voltammetry (LSV) curves in Supplementary Figs. 6, 7, respectively. Catalysts obtained under the optimized conditions were used for subsequent characterization. As shown in the above transmission electron microscopy (TEM) characterizations, ammonia treatment created an abundance of nanoholes, defects, and N-dopants in GNRs, which can facilitate mass transfer in the stacked catalyst layer and introduce significant pseudocapacitance ${ }^{30,31}$. Moreover, CNT backbones also improved the capacitances of GNR@CNT and N-GNR@CNT, compared with GNR and N-GNR, by alleviating stacking of GNRs (Supplementary Fig. 8) ${ }^{32,33}$. Benefitting from the ammonia treatment and CNT backbones, N-doped catalysts presented higher ORR activities than the non-doped counterparts; GNR@CNT and N-GNR@CNT also exhibited superior ORR activities in comparison to GNR and N-GNR
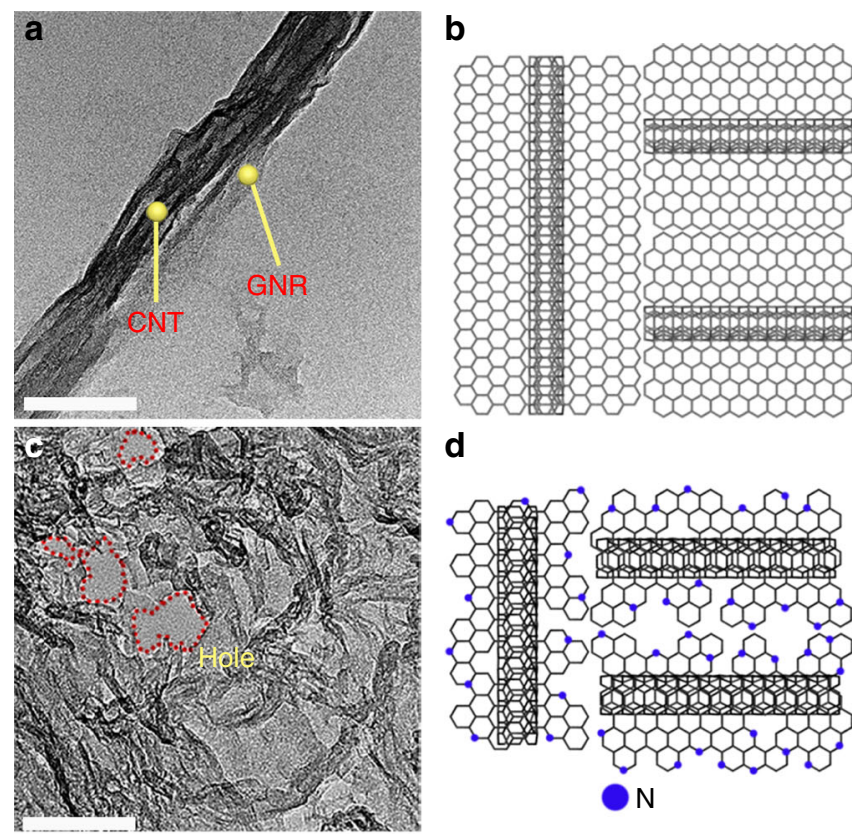

d
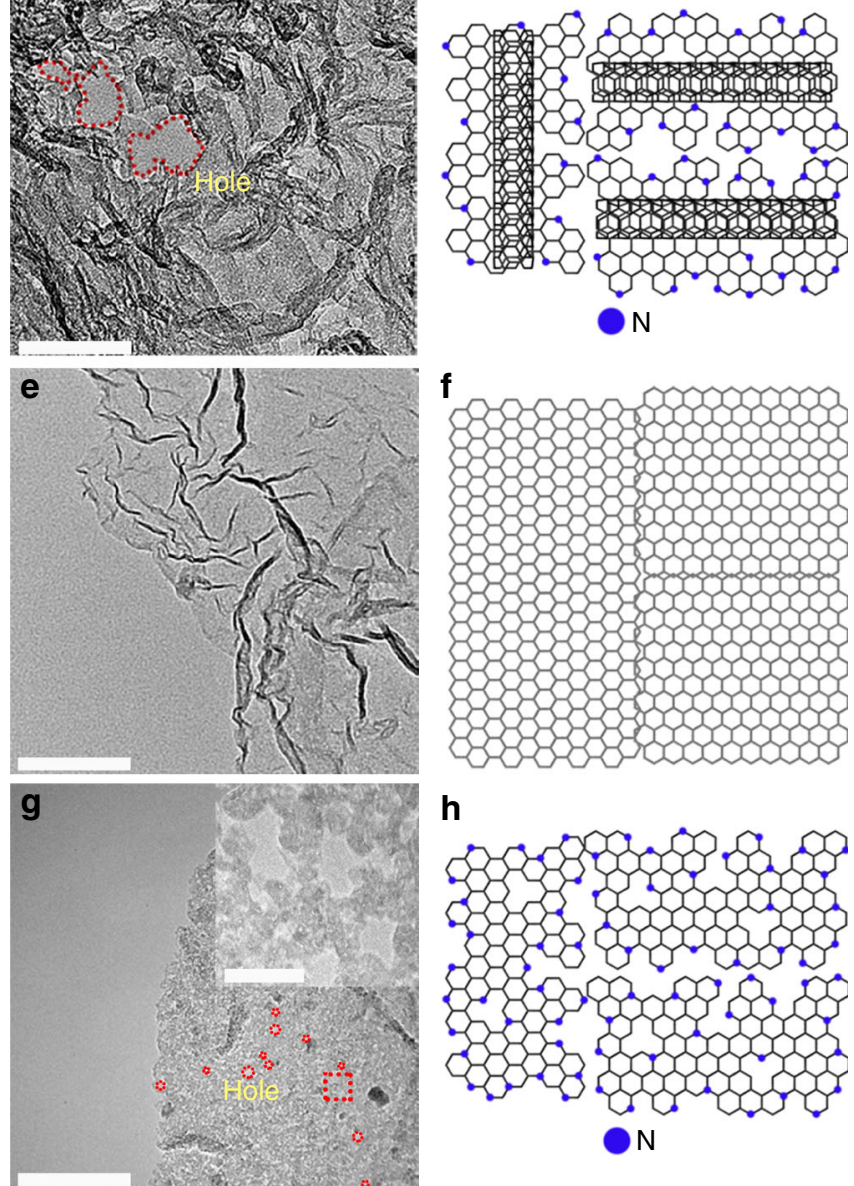

h

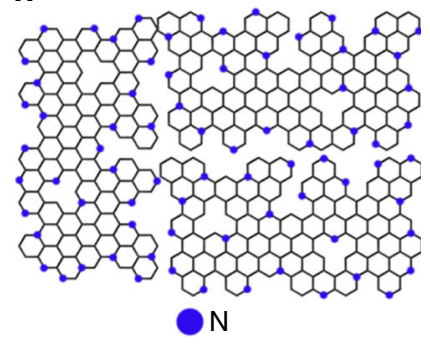

Fig. 2 Transmission electron microscopy images and schematic diagrams a, b Partially unzipping multiwall carbon nanotube (MWCNT) to graphene nanoribbons on carbon nanotube (GNR@CNT), c, d nitrogen-doped GNR@CNT (N-GNR@CNT), e, f totally unzipped MWCNT to graphene nanoribbons (GNR), and $\mathbf{g}$, h nitrogen-doped GNR (N-GNR). Scale bar: 100 $\mathrm{nm}$, and $10 \mathrm{~nm}$ for inset micrograph in $\mathbf{g}$

counterparts in an alkaline electrolyte (Fig. 3a). It is worth noting that GNR@CNT displayed a high onset potential of $0.960 \mathrm{~V}$ and a half-wave potential of $0.819 \mathrm{~V}$, which were only a little less than those of N-GNR@CNT (0.990 and 0.839 V) and even close to those of $\mathrm{Pt} / \mathrm{C}(20 \mathrm{wt} \% \mathrm{Pt}, 1.030$ and $0.841 \mathrm{~V})$. As shown in Supplementary Fig. 9, all these metal-free catalysts had a low $\mathrm{H}_{2} \mathrm{O}_{2}$ yield $(<5.9 \%)$ and a high electron transfer number $(n=$ 3.87-3.96), indicating a $4 \mathrm{e}^{-}$ORR process in the alkaline electrolyte.

To further investigate the ORR mechanism, we carried out peroxide reduction reaction (PRR) tests for GNR@CNT, NGNR@CNT (Fig. 3b), and Pt/C (Supplementary Fig. 10) ${ }^{34}$. It is 

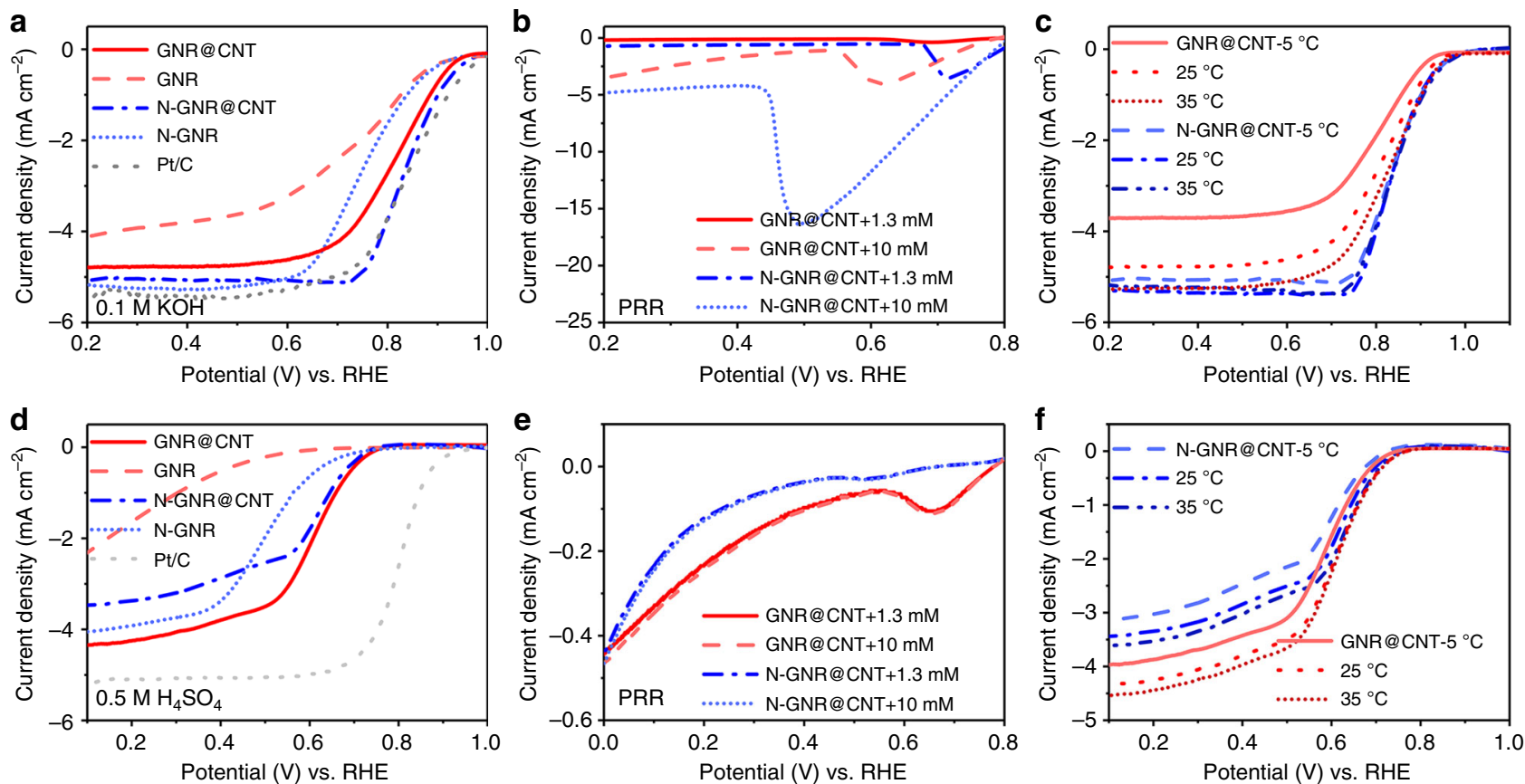

Fig. 3 Half-cell characterization of the catalysts. Linear sweep voltammetry curves of graphene nanoribbons on carbon nanotubes (GNR@CNT), N-doped GNR@CNT (N-GNR@CNT), and N-doped graphene nanoribbons (N-GNR) for a oxygen reduction reaction (ORR) activity, b peroxide reduction reaction (PRR) activity with 1.3 or $10 \mathrm{mM} \mathrm{H}_{2} \mathrm{O}_{2}$, and c ORR activity at 5,25 , and $35^{\circ} \mathrm{C}$ in $0.1 \mathrm{M} \mathrm{KOH}$; d ORR activity, e PRR activity with 1.3 or 10 mM $\mathrm{H}_{2} \mathrm{O}_{2}$, and f ORR activity at 5,25 , and $35^{\circ} \mathrm{C}$ in $0.5 \mathrm{M} \mathrm{H}_{2} \mathrm{SO}_{4}$. Electrolyte was $\mathrm{O}_{2}$-saturated, except for PRR experiments with Ar-saturated electrolyte. Rotating speed: $1600 \mathrm{rpm}$. Scan rate: $10 \mathrm{mV} \mathrm{s}^{-1}$

clear that the N-GNR@CNT and Pt/C showed high PRR activity, whereas the GNR@CNT displayed very low PRR activity at $\mathrm{H}_{2} \mathrm{O}_{2}$ concentrations of $1.3 \mathrm{mM}$ (the same concentration of saturated $\mathrm{O}_{2}$ in the electrolyte for the normal ORR test) and $10 \mathrm{mM}$. It suggests that the zigzag carbon did not prefer a " $2 \mathrm{e}^{-}+2 \mathrm{e}^{- \text {" ORR }}$ process $\left(\mathrm{O}_{2} \rightarrow \mathrm{H}_{2} \mathrm{O}_{2} \rightarrow \mathrm{H}_{2} \mathrm{O}\right)$. Therefore, a direct $4 \mathrm{e}^{-}$mechanism should dominate the ORR process of the GNR@CNT (zigzag carbon) in the alkaline electrolyte. The temperature dependence of catalytic activity was investigated for GNR@CNT and NGNR@CNT in the range of $5-35^{\circ} \mathrm{C}$. As presented in Fig. 3c, the half-wave potential and the limiting current density of GNR@CNT increased with increasing operation temperature. In contrast, N-GNR@CNT showed an insensitivity to the temperature. Additionally, the GNR and N-GNR displayed the similar temperature dependence as GNR@CNT and N-GNR@CNT, respectively (Supplementary Fig. 11), further confirming the distinctively different active sites between GNR@CNT and NGNR@CNT.

Our carbon catalysts were further characterized in an acid electrolyte, i.e. $0.5 \mathrm{M} \mathrm{H}_{2} \mathrm{SO}_{4}$. The capacitance tests in Supplementary Fig. 12 also confirmed the effects of ammonia treatment and CNT backbones, like in the alkline media. As shown in Fig. 3d, GNR@CNT exhibited a considerable activity with an onset potential of $0.760 \mathrm{~V}$ and a half-wave potential of $0.633 \mathrm{~V}$ although still lower than $\mathrm{Pt} / \mathrm{C}(20 \mathrm{wt} \% \mathrm{Pt})$ in the acid electrolyte $^{35}$. It is worth noting that both onset potential and limiting current of GNR@CNT were higher than those of NGNR@CNT in acid, which was opposite to the tendency in alkaline media. This phenomenon could be explained by the protonation of pyridinic nitrogen of N-GNR@CNT in the acid solution. As previously reported, the pyridinic nitrogen could adsorb proton in acidic medium, which reduced the affinity of $\mathrm{O}_{2}$ to the active carbon atoms adjacent to the pyridinic $\mathrm{N}$, thus decreasing the ORR activity of N-GNR@CNT ${ }^{36,37}$. In contrast, the GNR@CNT could be tolerant to the protonation reaction.
The electron transfer number of GNR@CNT was 3.6-3.9, suggesting the formation of $\mathrm{H}_{2} \mathrm{O}_{2}$ byproduct (Supplementary Fig. 13). Different from the previous PRR results in the alkaline electrolyte (Fig. 3b), both GNR@CNT and N-GNR@CNT showed quite weak PRR currents $\left(<0.4 \mathrm{~mA} \mathrm{~cm}^{-2}\right)$ no matter with a high or low concentration of $\mathrm{H}_{2} \mathrm{O}_{2}$. This phenomenon indicates that GNR@CNT and N-GNR@CNT had no activity to PRR in the acid electrolyte, which differed from the Pt/C catalyst (Supplementary Fig. 14). Therefore, both GNR@CNT and N-GNR@CNT should undergo a direct $4 \mathrm{e}^{-}$ORR with a negligible " $2 \mathrm{e}^{-}+2 \mathrm{e}^{- \text {" }}$ process in acid. The temperature dependence of electrocatalytic activities for GNR@CNT, N-GNR@CNT, GNR, and N-GNR was illustrated in Fig. 3f and Supplementary Fig. 15-16. All these catalysts displayed increased ORR activities with increasing temperature from 5 to $35^{\circ} \mathrm{C}$. Since the practical PEMFCs are usually operated at tens of degrees Centigrade, this temperature dependence implied a promising future for metal-free electrocatalysts to achieve high PEMFC performance ${ }^{38}$.

After the half-cell characterization, GNR@CNT, GNR, N-GNR@CNT, and N-GNR were assembled into the membrane electrode assembly (MEA) for evaluation in an actual PEMFC. Per our experience, the well separation of GNR in the catalyst layer should be crucial for a good PEMFC performance. Here carbon black (XC-72) was added into the catalyst layer as a spacer to separate GNR. GNR@CNT exhibited the best polarization curve and the highest peak power density of $520 \mathrm{~W} \mathrm{~g}^{-1}$, better than $430 \mathrm{~W} \mathrm{~g}^{-1}$ of N-GNR@CNT at the catalyst loading of $0.25 \mathrm{mg} \mathrm{cm}^{-2}$ (Fig. 4a). N-GNR and GNR presented relatively poor polarization curves and low power performances $\left(<200 \mathrm{~W} \mathrm{~g} \mathrm{~g}^{-1}\right)$ due to the lack of CNT backbones. To the best our knowledge, the obtained gravimetric peak power density of GNR@CNT in PEMFC is the highest value among all reported metal-free electrocatalysts and comparable to most NPMCs (Supplementary Table S4). Moreover, we found a strong dependence of fuel cell performance on catalyst loading. When 

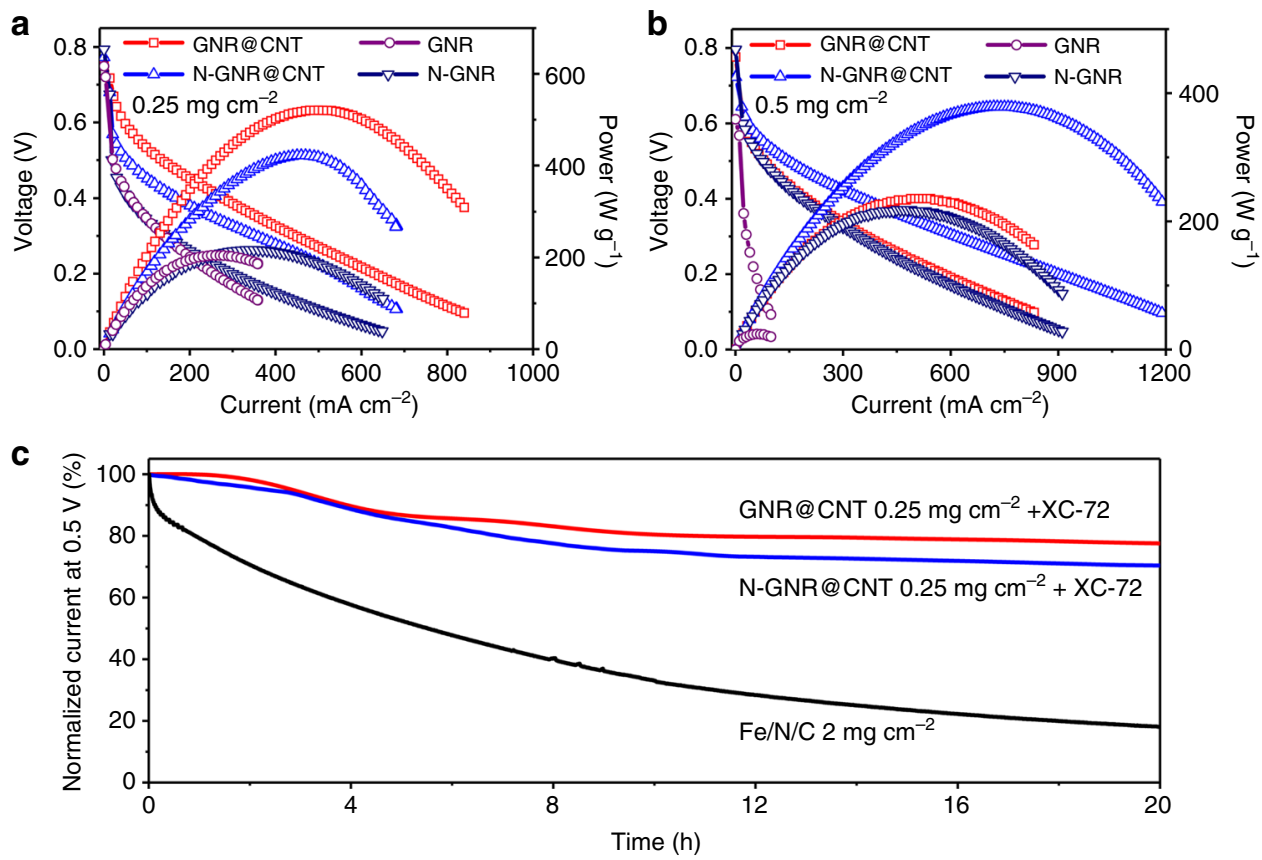

Fig. 4 Proton exchange membrane fuel cell evaluation. Polarization and power density curves of graphene nanoribbons on carbon nanotubes (GNR@CNT), N-doped GNR@CNT (N-GNR@CNT), and N-doped graphene nanoribbons (N-GNR) as a function of the areal current density with cathode catalyst loading of $\mathbf{a} 0.25 \mathrm{mg} \mathrm{cm}^{-2}$ and $\mathbf{b} 0.50 \mathrm{mg} \mathrm{cm}^{-2}$ in a proton exchange membrane fuel cell (PEMFC); c stability of the indicated catalysts in PEMFC measured at $0.5 \mathrm{~V}$. The absolute current densities before durability tests (at 100\%) were 136, 80 and $1216 \mathrm{~mA} \mathrm{~cm}^{-2}$ for graphene nanoribbons on carbon nanotube (GNR@CNT), nitrogen-doped catalyst (N-GNR@CNT), and reference catalyst iron-nitrogen-carbon (Fe/N/C), respectively. Weight ratio of Nafion/ catalyst/carbon black $(X C-72)=5 / 1 / 4$. Cell: $80^{\circ} \mathrm{C} ; \mathrm{H}_{2} / \mathrm{O}_{2}: 80^{\circ} \mathrm{C}, 100 \%$ relative humidity, 2 bar back pressure

the catalyst loading was doubled to $0.5 \mathrm{mg} \mathrm{cm}^{-2}$, the power density of GNR@CNT declined obviously (Fig. 4b), due to the worsened oxygen transport in the thick catalyst layer. For $\mathrm{N}$ GNR@CNT, the performance decay was relatively small in comparison with GNR@CNT, which could be ascribed to the nanoholes on N-GNR@CNT (Fig. 2b, c) to facilitate the mass transport in the catalyst layer ${ }^{39}$. When expressed in the unit of $\mathrm{W}$ $\mathrm{cm}^{-2}$, GNR@CNT and N-GNR@CNT could reach 161 and 241 $\mathrm{mW} \mathrm{cm}{ }^{-2}$, respectively (Supplementary Fig. 17), which are among the top performance metal-free catalysts ${ }^{16,40}$. It should be noted that the open circuit voltage of nanoribbon catalysts as shown in Supplementary Table 5 were still less than that of $\mathrm{Pt} / \mathrm{C}$ and most NPMCs. Finally, GNR@CNT, N-GNR@CNT, and a representative $\mathrm{Fe} / \mathrm{N} / \mathrm{C}$ catalyst were subject to a stability test in PEMFC at a constant voltage of $0.5 \mathrm{~V}$ with pure $\mathrm{H}_{2} / \mathrm{O}_{2}$ as fuel gases. As shown in Fig. 4c, N-GNR@CNT exhibited a more stable current performance than that of $\mathrm{Fe} / \mathrm{N} / \mathrm{C}$, which was consistent with previous reports ${ }^{16,41}$. It is interesting to find that the GNR@CNT possessed an even better PEMFC stability than NGNR@CNT at $0.5 \mathrm{~V}$. To the best of our knowledge, this is the only zigzag carbon that exhbits both high activity and stability in a PEMFC.

The observed high PEMFC performance prompted us to identify the active sites of GNR@CNT. Since both the defective carbon atoms and the oxidized defect carbon atoms are active to $\operatorname{ORR}^{19,42}$ and defective carbon atoms could be oxidized after long exposure to air, we prepared a couple of samples with controlled oxidative defects as active sites. GNR@CNT was protected under Ar atmosphere during the synthesis, storage, and ink preparation, while the control sample was exposed to air for 5 days (denoted as GNR@CNT-5days) after the synthesis to enhance the oxidation ${ }^{43}$. It has been previously reported that the enhanced oxidation on CNT could decrease the $s p^{2}$ peak and increase the $s p^{3}$ peak in the
C $1 s$ XPS spectrum, accompanied by a hydroxyl group $(\mathrm{C}-\mathrm{OH})$ transferring to a carboxyl group $(\mathrm{O}=\mathrm{C}-\mathrm{O})$ and then to $\mathrm{CO}_{2}$ and $\mathrm{H}_{2} \mathrm{O}^{44,45}$. The decreased $s p^{2} / s p^{3}$ ratio and increased $\mathrm{O}=\mathrm{C}-\mathrm{O} /$ $\mathrm{H}-\mathrm{O}-\mathrm{H}$ groups indicated a successful oxidation of GNR@CNT5days, as shown in Supplementary Fig. 18 and Table 1. In the acid electrolyte, the ORR activity of GNR@CNT-5days was obviously lower than that of GNR@CNT in terms of half-wave potential, current density, electron transfer number, and $\mathrm{H}_{2} \mathrm{O}_{2}$ yield, as shown in Supplementary Fig. 19. Hence, the observed high ORR activity of GNR@CNT in PEMFC should be mainly ascribed to the zigzag carbon rather than the oxidized zigzag carbon.

Theoretical calculations of free energy. To further identify the active site on GNR in the acid electrolyte, a DFT method was employed to calculate the free energy of ORR process on the representative carbon atoms. Although zigzag edges of GNR@CNT could be decorated by a small amount of -O (or $-\mathrm{OH})$, the $\mathrm{OH}-\mathrm{C}$ could be excluded since it could not adsorb $\mathrm{O}_{2}$ for $\mathrm{ORR}^{42}$. So, five types of carbon atoms on GNR were deduced as possible active sites, including (a) zigzag carbon atom, (b) carbon atom in basal plane, (c) carbon atom near O-doped zigzag edge, (d) carbon atom at armchair edge, and (e) carbon atom near a void as shown in Fig. 5. The minimum energy pathways for ORR on each type of carbon atoms were calculated (for more computational details see Supporting Information). As shown in Fig. $5 \mathrm{a}$, the most active site under $\mathrm{pH}=0.25\left(0.5 \mathrm{M} \mathrm{H}_{2} \mathrm{SO}_{4}\right)$ was figured out to be the zigzag carbon atom (a) (see Supplementary Fig. 20 for ORR process on zigzag carbon), which possessed the smallest limiting $\Delta \mathrm{G}_{\mathrm{OOH}^{\star}}$ of $0.54 \mathrm{eV}$ for $\mathrm{O}_{2}{ }^{\star}$ to $\mathrm{OOH}^{\star}$ at $U_{\mathrm{NHE}}=$ $0.745 \mathrm{~V}$ (onset potential for GNR@CNT in $0.5 \mathrm{M} \mathrm{H}_{2} \mathrm{SO}_{4}$, ${ }^{*}$ stands for an active site on the catalyst). The obtained $\Delta \mathrm{G}_{\mathrm{OOH}^{*}}$ is very close to the reported value for pure graphene in $0.1 \mathrm{M} \mathrm{KOH}^{46}$. In contrast, the carbon atom in GNR basal plane, the carbon atom 
a
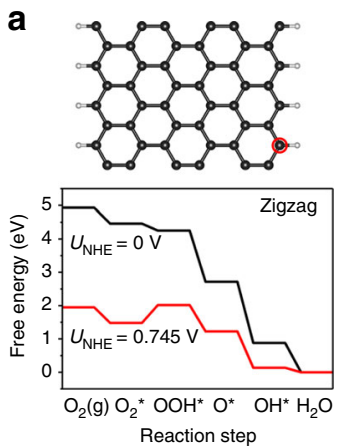

b
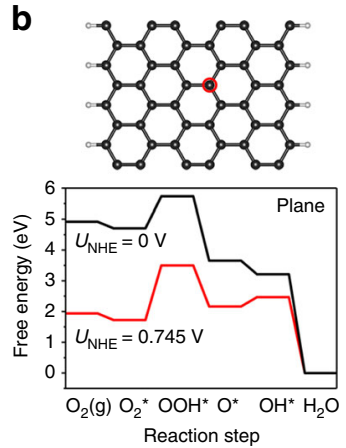

c
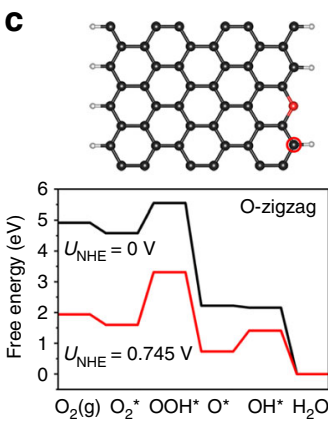

Reaction step d
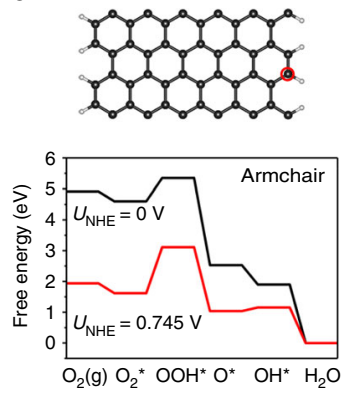

Reaction step e
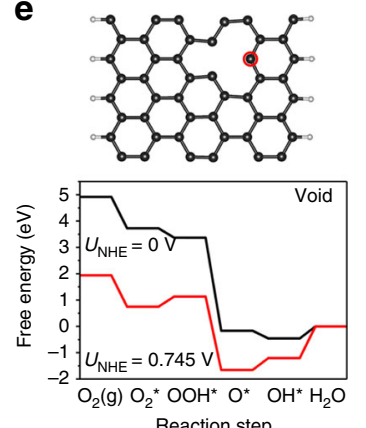

Reaction step

Fig. 5 Theoretical calculations. Models (top) and the corresponding free energy diagrams (bottom) for cycled carbon atoms at electrode potential $U_{\mathrm{NHE}}=$ 0 and $0.745 \mathrm{~V}$ in $0.5 \mathrm{M} \mathrm{H}_{2} \mathrm{SO}_{4}\left(\mathrm{NHE}\right.$ normal hydrogen electrode, $\mathrm{RHE}$ reversible hydrogen electrode, $\left.U_{\mathrm{NHE}}=U_{\mathrm{RHE}}-0.0591 \times \mathrm{pH}, \mathrm{pH}=0.25\right)$. a Carbon atom at zigzag edge, $\mathbf{b}$ carbon atom in basal plane, $\mathbf{c}$ carbon atom at O-doped zigzag edge, $\mathbf{d}$ carbon atom at armchair edge, and $\mathbf{e}$ carbon atom near a void

near O-doped zigzag edge, and the armchair carbon had significantly enlarged free energy for the step of $\mathrm{O}_{2}^{*}$ to $\mathrm{OOH}^{*}$, and the corresponding $\Delta G$ were $1.78,1.71$, and $1.46 \mathrm{eV}$, respectively (Fig. $5 \mathrm{~b}-\mathrm{d}$ ). As to the carbon atom near a void, the rate-limiting $\Delta G$ was $1.24 \mathrm{eV}$ for the step of $\mathrm{OH}^{*}$ to $\mathrm{H}_{2} \mathrm{O}$. These large $\Delta G$ hindered the formation of $\mathrm{OOH}^{\star}$ (the key intermediate) or $\mathrm{H}_{2} \mathrm{O}$, and thus excluded their possibility as ORR active sites at the onset potential.

\section{Discussion}

In summary, the zigzag-edged GNRs with CNT backbones (GNR@CNT) have been developed by partially unzipping MWCNTs and used as the metal-free ORR electrocatalyst for the $\mathrm{H}_{2} / \mathrm{O}_{2}$ PEMFCs. With the assistance of a CNT backbone and carbon black spacer, the mass transport in the catalyst layer was enhanced, coupled with an enhanced exposure of the zigzag carbon active sites along the GNR edge. Among all carbon-based metal-free electrocatalysts, GNR@CNT achieved the highest gravimetric power density of $520 \mathrm{~W} \mathrm{~g} \mathrm{~g}^{-1}$ in PEMFCs to the best of our knowledge. More importantly, GNR@CNT demonstrated an improved stability compared to a Fe/N/C catalyst in PEMFCs. DFT calculations coupled with experimental results revealed that zigzag carbon atoms possessed the higher electrocatalytic activity to ORR, than oxidized zigzag carbon, basal plane carbon, armchair edge carbon, and carbon atom near a void in acid electrolyte. This study demonstrates a great potential for defective graphitic carbon to be used in PEMFC application.

\section{Methods \\ Reagents. MWCNTs (length: $0.5-2 \mu \mathrm{m}$, outer diameter: $10-20 \mathrm{~nm}$ ) were obtained from Chengdu Organic Chemicals Co. Ltd. (Chengdu). Concentrated sulfuric acid, concentrated hydrochloric acid, potassium permanganate, and hydrogen peroxide were obtained from Sinopharm Chemical Reagent Beijing Co. Ltd. (Beijing). Pt/C $(20 \mathrm{wt} \% \mathrm{Pt})$ and $\mathrm{Pt} / \mathrm{C}(40 \mathrm{wt} \% \mathrm{Pt})$ were purchased from Sigma-Aldrich. Besides CNTs, all of the other materials were of analytical grade and used without further purification.}

Preparation of catalysts. To purify nanotubes (to remove metal impurities), raw MWCNT was stirred in concentrated $\mathrm{HCl}$ for $24 \mathrm{~h}$. A unit of $200 \mathrm{mg}$ purified MWCNT was put into a flask and mixed with $36 \mathrm{~mL}$ concentrated $\mathrm{H}_{2} \mathrm{SO}_{4}$. After $30 \mathrm{~min}$ of ultra-sonication and $1 \mathrm{~h}$ stirring, $800 \mathrm{mg} \mathrm{KMnO}_{4}(1600 \mathrm{mg}$ for completed unzipping) was slowly added into the mixture and stirred for $1 \mathrm{~h}$. The mixture was further heated in oil bath at $55^{\circ} \mathrm{C}$ for $15 \mathrm{~min}$ and then at $70{ }^{\circ} \mathrm{C}$ for 45 $\mathrm{min}$. After the mixture cooled down to the room temperature, it was poured into $100 \mathrm{~mL}$ ice water (containing $3 \mathrm{~mL} 30 \mathrm{wt} \% \mathrm{H}_{2} \mathrm{O}_{2}$ ) and then washed in $10 \mathrm{wt} \% \mathrm{HCl}$ solution five times and collected by centrifuge at $12000 \mathrm{rpm}$, and finally went through a dialysis for 1 week. Sample solution was freeze-dried and annealed in $\mathrm{Ar}$ atmosphere at $900^{\circ} \mathrm{C}$ for $30 \mathrm{~min}$ for defective carbon GNR@CNT and GNR, or in $\mathrm{NH}_{3}$ atmosphere at $800^{\circ} \mathrm{C}$ for 30 min for N-doped catalysts N-GNR@CNT and N-GNR.

$\mathrm{Fe} / \mathrm{N} / \mathrm{C}$ was synthesized according to literature ${ }^{47}$. Specifically, we performed ball milling on $100 \mathrm{mg}$ zeolitic imidazolate framework (ZIF-8) together with $10 \mathrm{mg}$ tris(1, 10-phenanthroline) iron(II) perchlorate ion for $1 \mathrm{~h}$, which was subsequently heated in $\mathrm{Ar}$ at $1000^{\circ} \mathrm{C}$ for $1 \mathrm{~h}$, and then at $900^{\circ} \mathrm{C}$ under $\mathrm{NH}_{3}$ for $15 \mathrm{~min}$.

Electrochemical measurements in half cell. Electrochemical properties were characterized using rotating ring disc electrode (model 3A, ALS Co.) in a threeelectrode beaker cell equipped with a Pt wire counter electrode and a saturated $\mathrm{Ag} /$ $\mathrm{AgCl}$ reference electrode. Here $0.1 \mathrm{M} \mathrm{KOH}$ or $0.5 \mathrm{M} \mathrm{H}_{2} \mathrm{SO}_{4}$ was used as the electrolyte. Before the electrochemical experiments, the $\mathrm{Ag} / \mathrm{AgCl}$ reference electrode was calibrated and potentials were converted to reversible hydrogen electrode (RHE) scale $\left(V_{\mathrm{RHE}}=V_{\mathrm{Ag} / \mathrm{AgCl}}+0.0591 \times \mathrm{pH}+0.197 \mathrm{~V}\right)$. The catalyst ink was prepared by dispersing $1 \mathrm{mg}$ catalyst in Nafion solution $\left(100 \mu \mathrm{L}, 1 \mathrm{mg} \mathrm{mL}^{-1}\right)$. The working electrode was prepared by dropping the catalyst ink $(5 \mu \mathrm{L})$ onto the glassy carbon disk electrode ( $4 \mathrm{~mm}$ diameter) and then drying at room temperature.

Loading amount of the catalysts was $398 \mu \mathrm{g} \mathrm{cm}{ }^{-2}$ (Pt/C (20 wt\% Pt) $\left.99 \mu \mathrm{g} \mathrm{cm}^{-2}\right)$. The LSV were recorded at a scan rate of $10 \mathrm{mV} \mathrm{s}^{-1}$ in an $\mathrm{O}_{2}$-saturated electrolyte (rotating speed: $1600 \mathrm{rpm}$, scan range: $0.2-1.1 V_{\mathrm{RHE}}$ ). The PRR polarization curves were recorded at $10 \mathrm{mV} \mathrm{s}^{-1}$ and $1600 \mathrm{rpm}$ rotation speeds in $\mathrm{Ar}$-saturated $0.1 \mathrm{M}$ $\mathrm{KOH}$ and $0.5 \mathrm{M} \mathrm{H}_{2} \mathrm{SO}_{4}$ solution with 1.3 and $10 \mathrm{mM} \mathrm{H}_{2} \mathrm{O}_{2}$ electrolytes. ORR and PRR results were presented after the subtraction of the capacitive background measured in Ar-saturated $0.1 \mathrm{M} \mathrm{KOH}$ or $0.5 \mathrm{M} \mathrm{H}_{2} \mathrm{SO}_{4}$ electrolyte.

The electron transfer number $(n)$ and $\mathrm{OOH}^{-}$intermediate production percentage $\left(\% \mathrm{OOH}^{-}\right)$were determined by:

$$
\begin{gathered}
n=4 \times \frac{I_{\mathrm{d}}}{I_{\mathrm{d}}+I_{\mathrm{r}} / N} \\
\% \mathrm{OOH}^{-}=200 \times \frac{I_{\mathrm{r}} / N}{I_{\mathrm{d}}+I_{\mathrm{r}} / N}
\end{gathered}
$$

where $I_{\mathrm{d}}$ is the disk current, $I_{\mathrm{r}}$ is the ring current, and $N$ is the current collection efficiency of the Pt ring, which was determined to be 0.4 .

PEMFC test. The synthesized catalyst was used as the cathode for a PEMFC. A typical catalyst ink was prepared by dispersing $1.25 \mathrm{mg}$ catalyst, $5 \mathrm{mg}$ XC-72, and $125 \mathrm{mg}$ Nafion solution (5 wt\% Nafion) in deionized water $(300 \mu \mathrm{L})$ and isopropanol $(600 \mu \mathrm{L})$ by sonication and stirring. The catalyst ink was coated on $5 \mathrm{~cm}^{2}$ carbon paper at a loading of $0.25 \mathrm{mg} \mathrm{cm}^{-2}$. For the anode, the catalyst was a commercial Pt/C (40 wt\% Pt) catalyst with a loading of $0.2 \mathrm{mg} \mathrm{Pt} \mathrm{per} \mathrm{cm}^{2}$. MEAs were fabricated by hot-pressing the anode, cathode, and a Nafion membrane (model: NRE 211) at $1.5 \mathrm{MPa}$ for $120 \mathrm{~s}$ at $130^{\circ} \mathrm{C}$. The performance of the fuel cell was assessed using a Model 850e fuel cell test system (Scribner Associates Inc.) operated at $80^{\circ} \mathrm{C}$. The $\mathrm{H}_{2}$ and $\mathrm{O}_{2}$ flow rates were 0.3 and $0.5 \mathrm{~L} \mathrm{~min}^{-1}$ at $100 \%$ relative humidity and 2 bar back pressure.

Computational methods. The DFT calculation was performed within a generalized gradient approximation designed by Perdew-Burke-Ernzerhof, as implemented in the Vienna ab initio simulation package. The projector-augmented wave method is used to describe the ionic potentials. The unit box was built for calculations with a volume of $9.86 \AA \times 29.00 \AA \times 18.00 \AA$ for zigzag carbon, basal plane carbon, oxidized zigzag carbon, and carbon near a void, or $8.54 \AA \times 29.00 \AA \times$ $18.00 \AA$ for armchair carbon. The plane wave basis set has a high cutoff energy of $500 \mathrm{eV}$ throughout the computation. The $K$-point sampling of the Brillouin zone was obtained using a $1 \times 1 \times 1$ grid generating meshes with their origin centered at the gamma $(\Gamma)$ point. All calculations were spin-polarized and the force convergence criterion for atomic relaxation was $0.01 \mathrm{eV}^{-1}$. The ORR can proceed either through a two-step two-electron pathway that reduces $\mathrm{O}_{2}$ to $\mathrm{H}_{2} \mathrm{O}_{2}$ or via a direct four-electron process in which $\mathrm{O}_{2}$ is directly reduced to $\mathrm{H}_{2} \mathrm{O}$ without involvement of an $\mathrm{H}_{2} \mathrm{O}_{2}$ intermediate. Here we study the complete reduction cycle 
because GNR@CNT shows a transfer number of about 3.7 in ORR, which is close to the four-electron process. In the acidic environment, the ORR can be written as:

$$
\begin{aligned}
\mathrm{O}_{2}(\mathrm{~g})+^{*} & \rightarrow \mathrm{O}_{2}^{*} \\
\mathrm{O}_{2}^{*}+\mathrm{H}^{+}+\mathrm{e}^{-} & \rightarrow \mathrm{OOH}^{*} \\
\mathrm{OOH}^{*}+\mathrm{H}^{+}+\mathrm{e}^{-} & \rightarrow \mathrm{O}^{*}+\mathrm{H}_{2} \mathrm{O}(1) \\
\mathrm{O}^{*}+\mathrm{H}^{+}+\mathrm{e}^{-} & \rightarrow \mathrm{OH}^{*} \\
\mathrm{OH}^{*}+\mathrm{H}^{+}+\mathrm{e}^{-} & \rightarrow \mathrm{H}_{2} \mathrm{O}(\mathrm{l})+^{*}
\end{aligned}
$$

where ${ }^{\star}$ stands for an active site on the graphene surface or at zigzag edge, (l) and (g) refer to liquid and gas phases, respectively, and $\mathrm{O}^{*}, \mathrm{OH}^{*}$, and $\mathrm{OOH}^{*}$ are adsorbed intermediates.

It was reported that the ORR rate-determining step can either be the $\mathrm{O}_{2}^{*}$ to $\mathrm{OOH}^{*}\left(\right.$ Eq. 4 ) or $\mathrm{OH}^{\star}$ to water (Eq. 7) ${ }^{48}$. The overpotential of the ORR processes can be determined by examining the reaction free energies $(\Delta G)$ of the different elementary steps. For each step the reaction free energy is defined as the difference between free energies of the initial and final states and is given by the expression:

$$
\Delta G=\Delta E+\Delta \mathrm{ZPE}-T \Delta S+\Delta G_{\mathrm{U}}+\Delta G_{\mathrm{pH}}
$$

Where $\Delta E$ is the reaction energy of reactant and product molecules adsorbed on catalyst surface obtained from DFT calculations. $\Delta G_{U}=-e U$, where $U$ is the potential at the electrode. $T$ is the temperature and $e$ is the transferred charge. $\triangle \mathrm{ZPE}$ is the change of zero-point energy computed by density functional perturbation theory. $\Delta S$ is the entropy change after the adsorption from the DFT simulation. $\Delta G_{\mathrm{pH}}$ (here $\mathrm{pH}$ is 0.25 ) is the correction of the $\mathrm{H}^{+}$free energy calculated by the concentration dependence of the entropy, in which $k_{\mathrm{B}}$ is the Boltzmann constant.

$$
\Delta G_{\mathrm{pH}}=-k_{\mathrm{B}} T \ln \left[\mathrm{H}^{+}\right]
$$

Physical characterization. The morphology and structure were characterized by TEM (JEM-2100F, Japan). XPS was performed on an ESCALAB 250Xi (Thermo Fisher Scientific Company, US) using a monochromated $\mathrm{Al} \mathrm{Ka}$ source and a pass energy of $50 \mathrm{eV}$ at a base pressure of $1 \times 10^{-8}$ mbar. FT-IR spectra were recorded using a Nicolet iN10 MX (Thermo Fisher Scientific Company).

\section{Data availability}

The data that support the findings of this study are available from the corresponding author upon reasonable request.

Received: 3 February 2018 Accepted: 27 August 2018

Published online: 19 September 2018

\section{References}

1. Chung, H. T. et al. Direct atomic-level insight into the active sites of a highperformance PGM-free ORR catalyst. Science 357, 479-484 (2017).

2. Zhu, Y. P., Guo, C., Zheng, Y. \& Qiao, S. Z. Surface and interface engineering of noble-metal-free electrocatalysts for efficient energy conversion processes. Acc. Chem. Res. 50, 915-923 (2017).

3. Yan, D. et al. Defect chemistry of nonprecious-metal electrocatalysts for oxygen reactions. Adv. Mater. 29, 1606459 (2017).

4. Liu, Q., Liu, X., Zheng, L. \& Shui, J. The solid-phase synthesis of an Fe-N-C electrocatalyst for high-power proton-exchange membrane fuel cells. Angew. Chem. Int. Ed. 57, 1204-1208 (2018).

5. Zhang, H. et al. Single atomic iron catalysts for oxygen reduction in acidic media: particle size control and thermal activation. J. Am. Chem. Soc. 139, 14143-14149 (2017)

6. Yang, L., Zeng, X., Wang, W. \& Cao, D. Recent progress in MOF-derived, heteroatom-doped porous carbons as highly efficient electrocatalysts for oxygen reduction reaction in fuel cells. Adv. Funct. Mater. 28, 1704537 (2017).

7. $\mathrm{Fu}, \mathrm{X}$. et al. In situ polymer graphenization ingrained with nanoporosity in a nitrogenous electrocatalyst boosting the performance of polymer-electrolytemembrane fuel cells. Adv. Mater. 29, 1604456 (2017).

8. Guo, D. et al. Active sites of nitrogen-doped carbon materials for oxygen reduction reaction clarified using model catalysts. Science 351, 361-365 (2016).
9. Yang, H. B. et al. Identification of catalytic sites for oxygen reduction and oxygen evolution in $\mathrm{N}$-doped graphene materials: development of highly efficient metal-free bifunctional electrocatalyst. Sci. Adv. 2, e1501122 (2016).

10. Li, J. C., Hou, P. X. \& Liu, C. Heteroatom-doped carbon nanotube and graphene-based electrocatalysts for oxygen reduction reaction. Small 13, 1702002 (2017).

11. Ricke, N. D. et al. Molecular-level insights into oxygen reduction catalysis by graphite-conjugated active sites. ACS Catal. 7, 7680-7687 (2017).

12. Sinthika, S., Waghmare, U. V. \& Thapa, R. Structural and electronic descriptors of catalytic activity of graphene-based materials: first-principles theoretical analysis. Small 14, 1703609 (2018).

13. Zeng, $\mathrm{X}$. et al. Single-atom to single-atom grafting of $\mathrm{Pt}_{1}$ onto $\mathrm{Fe}-\mathrm{N}_{4}$ center: $\mathrm{Pt}_{1} @ \mathrm{Fe}-\mathrm{N}-\mathrm{C}$ multifunctional electrocatalyst with significantly enhanced properties. Adv. Energy Mater. 8, 1701345 (2018).

14. Chenitz, R. et al. A specific demetalation of $\mathrm{Fe}-\mathrm{N}_{4}$ catalytic sites in the micropores of $\mathrm{NC} \_\mathrm{Ar}+\mathrm{NH}_{3}$ is at the origin of the initial activity loss of the highly active $\mathrm{Fe} / \mathrm{N} / \mathrm{C}$ catalyst used for the reduction of oxygen in PEM fuel cells. Energy Environ. Sci. 11, 365-382 (2018).

15. Armel, V. et al. Structural descriptors of zeolitic-imidazolate frameworks are keys to the activity of Fe-N-C catalysts. J. Am. Chem. Soc. 139, 453-464 (2017)

16. Shui, J., Wang, M., Du, F. \& Dai, L. N-doped carbon nanomaterials are durable catalysts for oxygen reduction reaction in acidic fuel cells. Sci. Adv. 1, e1400129 (2015).

17. Shen, A. et al. Oxygen reduction reaction in a droplet on graphite: direct evidence that the edge is more active than the basal plane. Angew. Chem. Int. Ed. 53, 10804-10808 (2014).

18. Waki, K. et al. Non-nitrogen doped and non-metal oxygen reduction electrocatalysts based on carbon nanotubes: mechanism and origin of ORR activity. Energy Environ. Sci. 7, 1950-1958 (2014).

19. Jiang, Y. et al. Significant contribution of intrinsic carbon defects to oxygen reduction activity. ACS Catal. 5, 6707-6712 (2015).

20. Benzigar, M. R. et al. Highly crystalline mesoporous $\mathrm{C}_{60}$ with ordered pores: a class of nanomaterials for energy applications. Angew. Chem. Int. Ed. 57, 569-573 (2018).

21. Seredych, M., Szczurek, A., Fierro, V., Celzard, A. \& Bandosz, T. J. Electrochemical reduction of oxygen on hydrophobic ultramicroporous polyHIPE carbon. ACS Catal. 6, 5618-5628 (2016).

22. Zhao, Y. et al. Graphitic carbon nitride nanoribbons: graphene-assisted formation and synergic function for highly efficient hydrogen evolution. Angew. Chem. Int. Ed. 53, 13934-13939 (2014).

23. Tang, C. \& Zhang, Q. Nanocarbon for oxygen reduction electrocatalysis: dopants, edges, and defects. Adv. Mater. 29, 1604103 (2017).

24. Jiao, L., Zhang, L., Wang, X., Diankov, G. \& Dai, H. Narrow graphene nanoribbons from carbon nanotubes. Nature 458, 877-880 (2009).

25. Kosynkin, D. V. et al. Longitudinal unzipping of carbon nanotubes to form graphene nanoribbons. Nature 458, 872-876 (2009).

26. Kim, K., Sussman, A. \& Zettl, A. Graphene nanoribbons obtained by electrically unwrapping carbon nanotubes. ACS Nano 4, 1362-1366 (2010).

27. Elias, A. L. et al. Longitudinal cutting of pure and doped carbon nanotubes to form graphitic nanoribbons using metal clusters as nanoscalpels. Nano Lett. 10, 366-372 (2010).

28. Rangel, N. L., Sotelo, J. C. \& Seminario, J. M. Mechanism of carbon nanotubes unzipping into graphene ribbons. J. Chem. Phys. 131, 031105 (2009).

29. $\mathrm{Li}, \mathrm{X}$. et al. Simultaneous nitrogen doping and reduction of graphene oxide. J. Am. Chem. Soc. 131, 15939-15944 (2009).

30. Jeong, H. M. et al. Nitrogen-doped graphene for high-performance ultracapacitors and the importance of nitrogen-doped sites at basal planes. Nano Lett. 11, 2472-2477 (2011).

31. $\mathrm{Xu}, \mathrm{Y}$. et al. Holey graphene frameworks for highly efficient capacitive energy storage. Nat. Commun. 5, 4554 (2014).

32. Lin, L.-Y. et al. A novel core-shell multi-walled carbon nanotube@graphene oxide nanoribbon heterostructure as a potential supercapacitor material. $J$. Mater. Chem. A 1, 11237-11245 (2013).

33. Wang, J. et al. Nitrogen and sulfur co-doping of partially exfoliated MWCNTs as 3-D structured electrocatalysts for the oxygen reduction reaction. J. Mater. Chem. A 4, 5678-5684 (2016).

34. Choi, C. H. et al. Unraveling the nature of sites active toward hydrogen peroxide reduction in Fe-N-C catalysts. Angew. Chem. Int. Ed. 56, 8809-8812 (2017).

35. Zhang, J., Zhao, Z., Xia, Z. \& Dai, L. A metal-free bifunctional electrocatalyst for oxygen reduction and oxygen evolution reactions. Nat. Nanotechnol. 10, 444-452 (2015).

36. Liu, G., Li, X. G. \& Popov, B. N. Stability study of nitrogen-modified carbon composite catalysts for oxygen reduction reaction in polymer electrolyte membrane fuel cells. ECS Trans. 25, 1251-1259 (2009).

37. Rauf, M. et al. Insight into the different ORR catalytic activity of Fe/N/C between acidic and alkaline media: protonation of pyridinic nitrogen. Electrochem. Commun. 73, 71-74 (2016). 
38. Fleige, M., Holst-Olesen, K., Wiberg, G. K. H. \& Arenz, M. Evaluation of temperature and electrolyte concentration dependent oxygen solubility and diffusivity in phosphoric acid. Electrochim. Acta 209, 399-406 (2016).

39. Shui, J. L., Chen, C., Grabstanowicz, L., Zhao, D. \& Liu, D. J. Highly efficient nonprecious metal catalyst prepared with metal-organic framework in a continuous carbon nanofibrous network. Proc. Natl Acad. Sci. USA 112, 10629-10634 (2015).

40. Ding, W. et al. Space-confinement-induced synthesis of pyridinic- and pyrrolic-nitrogen-doped graphene for the catalysis of oxygen reduction. Angew. Chem. Int. Ed. 52, 11755-11759 (2013).

41. Geng, D. et al. High oxygen-reduction activity and durability of nitrogendoped graphene. Energy Environ. Sci. 4, 760-764 (2011).

42. Liu, Z. et al. In situ exfoliated, edge-rich, oxygen-functionalized graphene from carbon fibers for oxygen electrocatalysis. Adv. Mater. 29, 16006207 (2017).

43. Miwa, R. H., Veiga, R. G. A. \& Srivastava, G. P. Structural, electronic, and magnetic properties of pristine and oxygen-adsorbed graphene nanoribbons. Appl. Surf. Sci. 256, 5776-5782 (2010).

44. Larciprete, R., Gardonio, S., Petaccia, L. \& Lizzit, S. Atomic oxygen functionalization of double walled C nanotubes. Carbon N. Y. 47, 2579-2589 (2009).

45. Wang, W.-H., Huang, B.-C., Wang, L.-S. \& Ye, D.-Q. Oxidative treatment of multi-wall carbon nanotubes with oxygen dielectric barrier discharge plasma. Surf. Coat. Technol. 205, 4896-4901 (2011).

46. Jiao, Y., Zheng, Y., Jaroniec, M. \& Qiao, S. Z. Origin of the electrocatalytic oxygen reduction activity of graphene-based catalysts: a roadmap to achieve the best performance. J. Am. Chem. Soc. 136, 4394-4403 (2014).

47. Proietti, E. et al. Iron-based cathode catalyst with enhanced power density in polymer electrolyte membrane fuel cells. Nat. Commun. 2, 416 (2011).

48. Calle-Vallejo, F. \& Koper, M. T. M. First-principles computational electrochemistry: a chievements and challenges. Electrochim. Acta 84, 3-11 (2012).

\section{Acknowledgements}

This work was supported by the National Thousand Talents Plan of China, the National Natural Science Foundation of China (Grant Nos. 21673014 and 51732002), the 111 project (B17002) funded by the Ministry of Education of China and the Fundamental Research Funds for the Central Universities of China.

\section{Author contributions}

L.X. and Q.L. synthesized the catalysts, prepared the fuel cell test materials and conducted fuel cell tests, and processed the data. X.L. and H.D. performed physical characterizations (XPS, FT-IR, and TEM). Y.L. and J.Shang contributed theoretical calculations., X.L., J.Shui, and L.D. wrote and edited the manuscript. All authors contributed to discussions about the results and the manuscript. The project was conceived and supervised by J.Shui.

\section{Additional information}

Supplementary Information accompanies this paper at https://doi.org/10.1038/s41467018-06279-x.

Competing interests: The authors declare no competing interests.

Reprints and permission information is available online at http://npg.nature.com/ reprintsandpermissions/

Publisher's note: Springer Nature remains neutral with regard to jurisdictional claims in published maps and institutional affiliations.

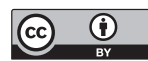

Open Access This article is licensed under a Creative Commons Attribution 4.0 International License, which permits use, sharing, adaptation, distribution and reproduction in any medium or format, as long as you give appropriate credit to the original author(s) and the source, provide a link to the Creative Commons license, and indicate if changes were made. The images or other third party material in this article are included in the article's Creative Commons license, unless indicated otherwise in a credit line to the material. If material is not included in the article's Creative Commons license and your intended use is not permitted by statutory regulation or exceeds the permitted use, you will need to obtain permission directly from the copyright holder. To view a copy of this license, visit http://creativecommons.org/ licenses/by/4.0/.

(C) The Author(s) 2018 